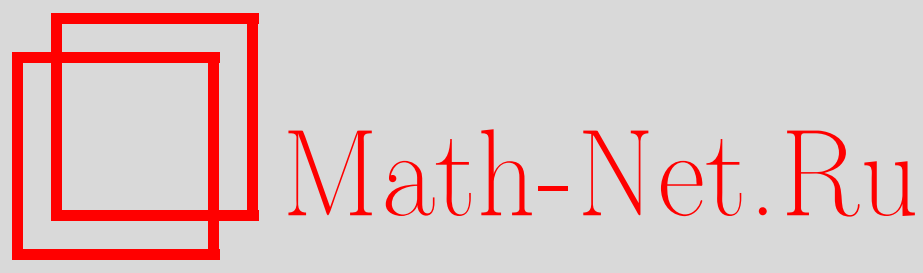

М. Е. Палистрант, Несоизмеримое состояние волны спиновой плотности и сверхпроводимость в квазидвумерных системах с анизотропным энергетическим спектром, ТМФ, 2011, том 168, номер 3, 503-517

DOI: https://doi.org/10.4213/tmf6697

Использование Общероссийского математического портала Math-Net.Ru подразумевает, что вы прочитали и согласны с пользовательским соглашением http://www.mathnet.ru/rus/agreement

Параметры загрузки:

IP : 54.84 .234 .179

26 апреля 2023 г., 09:43:12

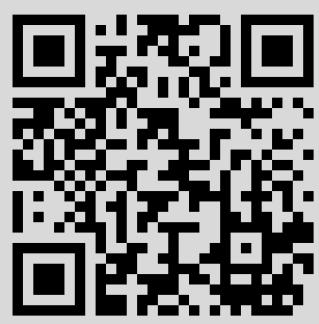




\section{НЕСОИЗМЕРИМОЕ СОСТОЯНИЕ ВОЛНЫ СПИНОВОЙ ПЛОТНОСТИ И СВЕРХПРОВОДИМОСТЬ В КВАЗИДВУМЕРНЫХ СИСТЕМАХ С АНИЗОТРОПНЫМ ЭНЕРГЕТИЧЕСКИМ СПЕКТРОМ}

Исследуются фазовые переходы в квазидвумерных системах с анизотропным энергетическим спектром и отклонением от серединного заполнения энергетической зоны $(\mu \neq 0)$. Показана возможность перехода диэлектрика в полуметаллическое состояние при нарушении условия нестинга из-за наличия параметра $\mu \neq 0$ и учета процессов переброса. Получены основные уравнения для параметров сверхпроводящего $(\Delta)$ и магнитного $(M)$ порядков, и определены условия зарождения сверхпроводимости на фоне состояния волны спиновой плотности, а также сосуществования сверхпроводимости и магнетизма. Показано, что переход магнитной системы в сверхпроводящее состояние с увеличением значения параметра $\mu$ при низких температурах может быть фазовым переходом первого рода. Получено также выражение для скачка теплоемкости $\left(C_{\mathrm{S}}-C_{\mathrm{N}}\right)$ при $T=T_{\mathrm{c}}$, зависящее от $M$ и $\mu$ и существенно отличающееся от случая теории Бардина-Купера-Шриффера. Уделяется внимание преобразованиям, связанным с плотностью электронных состояний рассматриваемой анизотропной системы, изменение которой может быть существенным под давлением или при легировании.

Ключевые слова: магнетизм, сверхпроводимость, термодинамические свойства.

90-летнему юбилею профессора С.В. Тябликова посвящается

\section{1. ВВЕДЕНИЕ}

Начиная с 2008 г. получено большое количество соединений на основе железа (iron-oxypnictides) с довольно высокой температурой сверхпроводящего перехода $T_{\text {c }}[1]-[3]$. В некоторых из этих соединений температура сверхпроводящего перехода достигает $55 \mathrm{~K}$ [4]. Это обстоятельство ставит их в один ряд с купратными сверхпроводящими при высоких температурах соединениями. Новые соединения демонстрируют существование на поверхности Ферми нескольких энергетических зон, как дырочных, так и электронных (вблизи поверхности Ферми эти зоны соответствуют $d$-состояниям $\mathrm{Fe}$ ), а также наличие условия нестинга, способствующего

* Институт прикладной физики АН Молдовы, Кишинев, Молдова.

E-mail: mepalistrant@yandex.ru 
возникновению антиферромагнитного состояния. Сверхпроводимость в этих соединениях возникает на фоне состояния волны спиновой плотности (ВСП) либо после подавления этого состояния. С общей точки зрения эта ситуация в случае сверхпроводимости не нова и в литературе известна [5]-[7]. История развития теории многозонных сверхпроводников изложена, например, в работах [8], [9].

В настоящее время возникает необходимость детального учета зонной структуры слоев FeAs [10]-[13]. Не исключено, что существенную роль в процессе возникновения магнетизма в этом слое играет обменное взаимодействие (стационарный магнетизм) наряду с состоянием ВСП [14]. При этом возникает спиральная структура антиферромагнитного состояния слоя FeAs. В этом плане, на наш взгляд, остаются актуальными и важными исследования профессора С. В. Тябликова, его идеи и методы в области магнетизма [15], [16].

Для объяснения свойств систем, в которых наблюдается сосуществование сверхпроводимости и состояния ВСП, предлагаются различные механизмы, связанные с нарушением условия нестинга, что приводит к неполной диэлектризации энергетического спектра [13], [17].

Наряду со сверхпроводящими при высоких температурах системами получено также много диэлектриков и полупроводников, в том числе с аналогичной зонной структурой. В ряде случаев эти диэлектрики и полупроводники под давлением становятся сверхпроводниками (см., например, работу [18]).

Отметим, что оксидные керамики и соединения на основе Fе обладают рядом одинаковых свойств (особенностей). В частности, и те и другие являются слоистыми структурами, что позволяет рассматривать их как квазидвумерные.

В настоящей работе мы отвлечемся от многозонности и уделим основное внимание состоянию ВСП (в соизмеримой и несоизмеримой фазах), а также возникновению сверхпроводимости на фоне магнетизма в двумерной системе с отклонением от серединного заполнения энергетической зоны $(\mu \neq 0)$ и с анизотропным энергетическим спектром. Анизотропия электронного энергетического спектра влияет на плотность электронных состояний $(N(\varepsilon))$, которая может существенно изменяться под влиянием давления или при легировании системы, изменяя, в свою очередь, сверхпроводящие и магнитные характеристики рассматриваемой системы.

Работа имеет следующую структуру. В разделе 2 приведен гамильтониан системы, выведены уравнения для электронных функций Грина и получена система уравнений, определяющая сосуществование состояния ВСП и сверхпроводимости. Выполнен переход от суммирования по $\vec{k}$ к интегрированию по энергии с учетом процессов переброса и возникшей несоизмеримости ВСП (волновой вектор $Q \neq 2 k_{\mathrm{F}}$ ). Раздел 3 посвящен преобразованию полученных уравнений для параметров порядка $\Delta$ и $M$ с целью выделения областей интегрирования по энергии, в которых $N(\varepsilon) \neq 0$. В разделе 4 рассматривается переход системы в несоизмеримое состояние ВСП и обсуждается возможность возникновения сверхпроводимости в магнитной фазе. Получена система уравнений, и найдены компоненты параметра порядка $\eta_{a}$ и $\eta_{b}$, определяющие наличие несоизмеримости ВСП, и критическое значение параметра $\mu=\mu_{\mathrm{c}}$, при котором эта несоизмеримость возникает. Также рассмотрены аналитически некоторые пределы, при которых оказалось возможным выяснить зарождение сверхпроводимости в несоизмеримой фазе ВСП. В разделе 5 вычислена разность свободных энергий и изучена область температур, близких к критической 
температуре сверхпроводящего перехода $T_{\mathrm{c}}$. Определены условия изменения типа фазового перехода, вычислен также скачок теплоемкости $\left.\frac{C_{\mathrm{S}}-C_{\mathrm{N}}}{N_{0}}\right|_{T=T_{\mathrm{c}}}$ и определена зависимость от $\mu$. В разделе 6 обсуждаются полученные в работе результаты.

\section{2. ГАМИЛЬТОНИАН СИСТЕМЫ И ОСНОВНЫЕ УРАВНЕНИЯ}

Рассматриваемая двумерная система описывается гамильтонианом

$$
\begin{aligned}
& H=\sum_{\vec{k}, \vec{\sigma}}(\varepsilon(\vec{k})-\mu) a_{\vec{k} \vec{\sigma}}^{+} a_{\vec{k} \vec{\sigma}}-\sum_{\vec{k}, \vec{k}^{\prime}} V\left(\vec{k} \vec{k}^{\prime}\right) a_{\vec{k} \uparrow}^{+} a_{-\vec{k} \downarrow} a_{-\vec{k}^{\prime} \downarrow} a_{\vec{k}^{\prime} \uparrow}+ \\
& \quad+\sum_{\vec{k}, \vec{k}^{\prime}, \vec{q}} I a_{\vec{k} \uparrow}^{+} a_{\vec{k}+\vec{q} \uparrow} a_{\vec{k}^{\prime} \downarrow}^{+} a_{\vec{k}^{\prime}-\vec{q} \downarrow},
\end{aligned}
$$

где $a_{\vec{k} \vec{\sigma}}^{+}, a_{\vec{k} \vec{\sigma}}-$ операторы рождения и уничтожения электронов (импульса $\vec{k}$ и спина $\vec{\sigma}), \mu$ - отклонение от серединного заполнения энергетической зоны. Первый член в этом выражении описывает кинетическую энергию электронов, второй член ответствен за сверхпроводимость, последний член определяет кулоновское взаимодействие электронов с противоположными спинами.

Учитывая в сумме по $\vec{q}$ значения $\vec{q}=0, \pm \vec{Q}$ и используя приближение среднего поля, представим гамильтониан (1) в виде

$$
H=\sum_{\vec{k}, \vec{\sigma}}(\varepsilon(\vec{k})-\mu) a_{\vec{k} \vec{\sigma}}^{+} a_{\vec{k} \vec{\sigma}}-\sum_{\vec{k}}\left(\Delta a_{\vec{k} \uparrow}^{+} a_{-\vec{k} \downarrow}+\text { э.c. }\right)-\sum_{\vec{k}, \vec{\sigma}}\left(\vec{\sigma} \vec{M} a_{\vec{k} \vec{\sigma}}^{+} a_{\vec{k}-\vec{Q} \vec{\sigma}}+\text { э.c. }\right),
$$

где $\Delta$ и $M=M_{z}$ - параметры сверхпроводящего и магнитного упорядочения соответственно. Величины $\Delta$ и $M$ определяются соотношениями

$$
\begin{aligned}
\Delta & =V T \sum_{\vec{k}} \sum_{\omega_{n}} F_{-\vec{k}, \vec{k}}^{\downarrow \uparrow}\left(\omega_{n}\right), \\
M & =\frac{I}{2} T \sum_{\vec{k}} \sum_{\omega_{n}} \sum_{\sigma} G_{\overrightarrow{\vec{k}}-\vec{Q}, \vec{k}}^{\vec{\sigma}}\left(\omega_{n}\right),
\end{aligned}
$$

где $V$ и $I$ - константы Бардина-Купера-Шриффера и магнитного взаимодействия соответственно, $F$ и $G^{\sigma \sigma}$ - фурье-образы температурных функций Грина, определяемых соотношениями

$$
\begin{aligned}
F_{\vec{k}, \vec{k}}^{\downarrow \uparrow}\left(\tau-\tau^{\prime}\right) & =\left\langle T_{\tau} a_{-\vec{k} \downarrow}^{+}(\tau) a_{\vec{k} \uparrow}^{+}\left(\tau^{\prime}\right)\right\rangle, \\
G_{\vec{k}-\vec{Q}, \vec{k}}^{\vec{\sigma}}\left(\tau-\tau^{\prime}\right) & =-\left\langle T_{\tau} a_{\vec{k}-\vec{Q} \vec{\sigma}}(\tau) a_{\vec{k} \vec{\sigma}}^{+}\left(\tau^{\prime}\right)\right\rangle,
\end{aligned}
$$

$\omega_{n}$ - мацубаровская частота. На основании гамильтониана (2) после преобразования Фурье уравнения движения для компонент электронных функций Грина можно представить в виде

$$
\begin{gathered}
\left(i \omega_{n}-\varepsilon_{1}\right) G_{\vec{k}, \vec{k}}^{\uparrow \uparrow}+\Delta F_{-\vec{k}, \vec{k}}^{\downarrow \uparrow}+M G_{\vec{k}-\vec{Q}, \vec{k}}^{\uparrow \uparrow}=1, \\
\Delta G_{\vec{k}, \vec{k}}^{\uparrow \uparrow}+\left(i \omega_{n}+\varepsilon_{1}\right) F_{-\vec{k}, \vec{k}}^{\downarrow \uparrow}+M F_{-\vec{k}+\vec{Q}, \vec{k}}^{\downarrow \uparrow}=0, \\
M G_{\vec{k}, \vec{k}}^{\uparrow \uparrow}+\left(i \omega_{n}-\varepsilon_{2}\right) G_{\vec{k}-\vec{Q}, \vec{k}}^{\uparrow \uparrow}+\Delta F_{\vec{k}+\vec{Q}, \vec{k}}^{\downarrow \uparrow}=0, \\
M F_{-\vec{k}, \vec{k}}^{\downarrow \uparrow}+\Delta G_{\vec{k}-\vec{Q}, \vec{k}}^{\uparrow \uparrow}+\left(i \omega_{n}+\varepsilon_{2}\right) F_{-\vec{k}+\vec{Q}, \vec{k}}^{\downarrow \uparrow}=0,
\end{gathered}
$$


где

$$
\varepsilon_{1}=\varepsilon(\vec{k})-\mu, \quad \varepsilon_{2}=\varepsilon(\vec{k}-\vec{Q})-\mu .
$$

Система уравнений (5) позволяет вычислить функции Грина $F_{-\vec{k}, \vec{k}}^{\downarrow \uparrow}\left(\omega_{n}\right)$ и $G_{\vec{k}-\vec{Q}, \vec{k}}^{\vec{\sigma}}\left(\omega_{n}\right)$, определяющие уравнения для параметров сверхпроводящего $(\Delta)$ и магнитного $(M)$ порядков. Получаем

$$
\begin{aligned}
F_{-\vec{k}, \vec{k}}^{\downarrow \uparrow}\left(\omega_{n}\right)= & \frac{\Delta\left(\omega_{n}^{2}+\varepsilon_{2}^{2}+\Delta^{2}-M^{2}\right)}{D\left(\omega_{n}\right)}, \\
G_{\vec{k}-\vec{Q}, \vec{k}}^{\vec{\sigma} \vec{\sigma}}\left(\omega_{n}\right)= & \sigma \frac{M\left[M^{2}-\Delta^{2}-\left(i \omega_{n}+\varepsilon_{1}\right)\left(i \omega_{n}+\varepsilon_{2}\right)\right]}{D\left(\omega_{n}\right)}, \\
D\left(\omega_{n}\right)= & \omega_{n}^{4}+\omega_{n}^{2}\left(\varepsilon_{1}^{2}+\varepsilon_{2}^{2}+2 M^{2}+2 \Delta^{2}\right)+\varepsilon_{1}^{2} \varepsilon_{2}^{2}-2 M^{2} \varepsilon_{1} \varepsilon_{2}+ \\
& +\Delta^{2}\left(\varepsilon_{1}^{2}+\varepsilon_{2}^{2}\right)+\left(M^{2}-\Delta^{2}\right)^{2} .
\end{aligned}
$$

Подставляя выражения для функций Грина (7) в соотношения (3), получаем

$$
\begin{gathered}
\Delta=V T \sum_{\vec{k}} \sum_{\omega_{n}} \frac{\Delta\left(\omega_{n}^{2}+\varepsilon_{2}^{2}+\Delta^{2}-M^{2}\right)}{D\left(\omega_{n}\right)}, \\
M=I \sum_{\vec{k}} T \sum_{\omega_{n}} \frac{M\left[M^{2}-\Delta^{2}-\left(i \omega_{n}+\varepsilon_{1}\right)\left(i \omega_{n}+\varepsilon_{2}\right)\right]}{D\left(\omega_{n}\right)} .
\end{gathered}
$$

Выполним суммирование по частоте $\omega_{n}=(2 n+1) \pi T, n= \pm 1, \pm 2, \ldots$, стандартным образом, используя соотношение

$$
T \sum_{n} F\left(i \omega_{n}\right)=\frac{1}{4 \pi i} \int_{C} F(z) \operatorname{th} \frac{\beta z}{2} d z,
$$

где контур $C$ охватывает полюсы функции $\operatorname{th}(\beta z / 2)$ в точках $z=i \omega_{n}$. Замыкая этот контур на бесконечности и выполнив интегрирование в комплексной плоскости, получаем

$$
\begin{aligned}
\frac{1}{V}=- & \frac{1}{2\left(X_{1}-X_{2}\right)} \sum_{\vec{k}}\left[-X_{1}+\varepsilon_{2}^{2}+\Delta^{2}-M^{2} \frac{\operatorname{th}\left(\beta \sqrt{X_{1}} / 2\right)}{\sqrt{X_{1}}}-\right. \\
& \left.-\left(-X_{2}+\varepsilon_{2}^{2}+\Delta^{2}-M^{2}\right) \frac{\operatorname{th}\left(\beta \sqrt{X_{2}} / 2\right)}{\sqrt{X_{2}}}\right], \\
\frac{1}{I}=- & \frac{1}{4} \sum_{\vec{k}} \frac{\operatorname{th}\left(\beta \sqrt{X_{1}} / 2\right)}{\sqrt{X_{1}}\left(X_{1}-X_{2}\right)}\left[2 M^{2}-2 \Delta^{2}-\left(\sqrt{X_{1}}+\varepsilon_{1}\right)\left(\sqrt{X_{1}}+\varepsilon_{2}\right)-\right. \\
& \left.-\left(\sqrt{X_{1}}-\varepsilon_{1}\right)\left(\sqrt{X_{1}}-\varepsilon_{2}\right)\right]- \\
& -\frac{\operatorname{th}\left(\beta \sqrt{X_{2}} / 2\right)}{\sqrt{X_{2}}\left(X_{1}-X_{2}\right)}\left[2 M^{2}-2 \Delta^{2}-\left(\sqrt{X_{2}}+\varepsilon_{1}\right)\left(\sqrt{X_{2}}+\varepsilon_{2}\right)\right],
\end{aligned}
$$

где

$$
\begin{gathered}
X_{1,2}=\frac{b \pm \sqrt{b^{2}-4 c}}{2}, \quad b=\varepsilon_{1}^{2}+\varepsilon_{2}^{2}+2 M^{2}-2 \Delta^{2}, \\
c=\varepsilon_{1}^{2} \varepsilon_{2}^{2}-2 M^{2} \varepsilon_{1} \varepsilon_{2}+\Delta^{2}\left(\varepsilon_{1}^{2}+\varepsilon_{2}^{2}\right)+\left(M^{2}-\Delta^{2}\right)^{2}, \\
X_{1}-X_{2}=\sqrt{b^{2}-4 c}, \quad b^{2}-4 c=\left(\varepsilon_{1}^{2}-\varepsilon_{2}^{2}\right)+4 M^{2}\left(\varepsilon_{1}+\varepsilon_{2}\right)^{2}+16 M^{2} \Delta^{2} .
\end{gathered}
$$


Мы выбираем анизотропный закон дисперсии энергии электронов

$$
\varepsilon(\vec{k})=\tilde{\varepsilon}_{1}-W_{1} \cos \left(k_{x} a\right)-W_{2} \cos \left(k_{y} b\right),
$$

в котором учтено взаимодействие ближайших соседей. Из (11) нетрудно видеть, что при $\mu=0$ и $q_{x}=q_{y}=0, Q_{x}^{0}=\pi / a, Q_{y}^{0}=\pi / b$ выполняется условие нестинга $\varepsilon(\vec{k})=-\varepsilon\left(\vec{k}-\vec{Q}^{0}\right)$. В этом случае возникает соизмеримое состояние ВСП и на поверхности Ферми появляется диэлектрическая щель. При получении уравнений для функций Грина (5) мы ограничились учетом функций $G_{k}, G_{k+Q}, F_{k}$, опуская функции $G_{k+2 Q}, F_{k+Q}$ и др. (одномерный случай см. в работах [19], [20]). Это можно сделать, если наложить ограничения

$$
\left|k_{x}-Q_{x}\right|<Q_{x}^{0}, \quad\left|k_{x}\right|<Q_{0} \quad \text { и } \quad\left|k_{y}-Q_{y}\right|<Q_{y^{\prime}}^{0}, \quad\left|k_{y}\right|<Q_{y}^{0},
$$

которые соответствуют электронно-дырочному спариванию в первой зоне Бриллюэна. На основании (12) имеем $q_{x}<k_{x}<Q_{x}^{0}$ и $q_{y}<k_{y}<Q_{y}^{0}$. Следовательно, в формулах (9) при учете только нормальных процессов суммирование по $\vec{k}$ следует выполнять в этих пределах. Учет процессов переброса сводится к суммированию по $\vec{k}$ в уравнениях (9) в интервале $-Q_{0}^{x}<k_{x}<Q_{0}^{x}$ и $-Q_{0}^{y}<k_{y}<Q_{0}^{y}$. Это значит, что вся зона Бриллюэна принимает участие в электронно-дырочном спаривании. Перейдем в формулах (9) от суммирования по $\vec{k}$ к интегрированию по формуле

$$
\sum_{\vec{k}} F\left(\varepsilon_{1} \varepsilon_{2}, \Delta, M\right)=\frac{1}{(2 \pi)^{2}} \int_{-Q_{0}^{x}}^{Q_{0}^{x}} d k_{x} \int_{-Q_{0}^{y}}^{Q_{0}^{y}} d k_{y} F\left(\varepsilon_{1}, \varepsilon_{2}, \Delta, M\right) .
$$

Вводя определения $\varepsilon_{1}=\varepsilon_{1 x}+\varepsilon_{1 y}-\mu$ и $\varepsilon_{2}=\varepsilon_{2 x}+\varepsilon_{2 y}-\mu$, где $\varepsilon_{1 x}=-W_{1} \cos \left(a k_{x}\right)$, $\varepsilon_{1 y}=-W_{2}\left(\cos \left(b k_{y}\right)\right), \varepsilon_{2 x}=-W_{1} \cos \left[a\left(k_{x}+q_{x}\right)\right], \varepsilon_{2 y}=-W_{2} \cos \left[b\left(k_{y}+q_{y}\right)\right]$, и обобщив методику преобразований, развитую в работах [21] для квазиодномерных систем (см. также работу [22] для квазидвумерных систем), приведем соотношение (13) к виду

$$
\sum_{\vec{k}} F\left(\varepsilon_{1} \varepsilon_{2}, \Delta, M\right)=\left.\frac{1}{4} \int_{-\widetilde{W}}^{\widetilde{W}} N(\varepsilon) \sum_{\alpha, \beta} F\left(\varepsilon_{1}, \varepsilon_{2}, \Delta, M\right)\right|_{\substack{\varepsilon_{1}=\varepsilon-\mu_{\beta}^{\alpha}, \varepsilon_{2}=-\left(\varepsilon+\mu_{\beta}^{\alpha}\right)}},
$$

где

$\widetilde{W}=W_{1}+W_{2}, \quad \mu_{\beta}^{\alpha}=\mu+\alpha \eta_{a}+\beta \eta_{b}, \quad \eta_{a}=\frac{W_{1} a q_{x}}{2}, \quad \eta_{b}=\frac{W_{2} b q_{y}}{2}, \quad \alpha, \beta= \pm 1$.

Перейдя в уравнениях для параметров порядка $\Delta$ и $M(9)$ от суммирования по $\vec{k}$ к интегрированию по энергии согласно формуле (14) и воспользовавшись определениями (10), приведем эти уравнения к виду

$$
\begin{aligned}
& \frac{1}{V}=\frac{1}{4} \int_{-\widetilde{W}}^{\widetilde{W}} d \varepsilon N(\varepsilon) \sum_{\alpha, \beta} \Phi_{1}\left(\varepsilon, \mu_{\beta}^{\alpha}, M, \Delta\right), \\
& \frac{1}{I}=\frac{1}{4} \int_{-\widetilde{W}}^{\widetilde{W}} d \varepsilon N(\varepsilon) \sum_{\alpha, \beta} \Phi_{2}\left(\varepsilon, \mu_{\beta}^{\alpha}, M, \Delta\right),
\end{aligned}
$$


где

$$
\begin{aligned}
\Phi_{1}\left(\varepsilon, \mu_{\beta}^{\alpha}, M, \Delta\right)= & \frac{M^{2}+\sqrt{\left(\mu_{\beta}^{\alpha}\right)^{2}\left(\varepsilon^{2}+M^{2}\right)+M^{2} \Delta^{2}}}{\sqrt{\left(\mu_{\beta}^{\alpha}\right)^{2}\left(\varepsilon^{2}+M^{2}\right)+M^{2} \Delta^{2}}} \frac{\operatorname{th}\left(\beta \sqrt{X_{1}} / 2\right)}{\sqrt{X_{1}}}- \\
& -\frac{M^{2}-\sqrt{\left(\mu_{\beta}^{\alpha}\right)^{2}\left(\varepsilon^{2}+M^{2}\right)+M^{2} \Delta^{2}}}{\sqrt{\left(\mu_{\beta}^{\alpha}\right)^{2}\left(\varepsilon^{2}+M^{2}\right)+M^{2} \Delta^{2}}} \frac{\operatorname{th}\left(\beta \sqrt{X_{2}} / 2\right)}{\sqrt{X_{2}}}, \\
\Phi_{2}\left(\varepsilon, \mu_{\beta}^{\alpha}, M, \Delta\right)= & \frac{\Delta^{2}+\left(\mu_{\beta}^{\alpha}\right)^{2}+\sqrt{\left(\mu_{\beta}^{\alpha}\right)^{2}\left(\varepsilon^{2}+M^{2}\right)+M^{2} \Delta^{2}}}{\sqrt{\left(\mu_{\beta}^{\alpha}\right)^{2}\left(\varepsilon^{2}+M^{2}\right)+M^{2} \Delta^{2}}} \frac{\operatorname{th}\left(\beta \sqrt{X_{1}} / 2\right)}{\sqrt{X_{1}}}- \\
X_{1,2}= & \varepsilon^{2}+\left(\mu_{\beta}^{\alpha}\right)^{2}+\sqrt{\left(\mu_{\beta}^{\alpha}\right)^{2}\left(\varepsilon^{2}+M^{2}\right)+M^{2} \Delta^{2}} \\
\sqrt{\left(\mu_{\beta}^{\alpha}\right)^{2}\left(\varepsilon^{2}+M^{2}\right)+M^{2} \Delta^{2}} & \frac{\operatorname{th} \sqrt{X_{2}}}{\sqrt{X_{2}}}
\end{aligned}
$$

\section{3. ПЛОТНОСТЬ ЭЛЕКТРОННЫХ СОСТОЯНИЙ. ПРЕОБРАЗОВАНИЕ УРАВНЕНИЙ ДЛЯ ПАРАМЕТРОВ ПОРЯДКОВ $\Delta$ И $M$}

В соответствии с определением плотность электронных состояний рассматриваемой системы представляется в виде

$$
N(\varepsilon)=\sum_{k_{x}, k_{y}} \delta(\varepsilon-\varepsilon(\vec{k}))
$$

где функция $\varepsilon(\vec{k})$ дана формулой (11). Подставив в выражение (19) формулу (11) и выполнив двойное интегрирование по $k_{x}$ и $k_{y}$, приведем плотность электронных состояний к виду [23], [24]

$$
N(\varepsilon)=\frac{1}{\pi^{2} a b}\left\{\frac{\theta\left[\left(\varepsilon-\tilde{\varepsilon}_{1}\right)^{2}-\left(W_{1}-W_{2}\right)^{2}\right]}{\sqrt{W_{1} W_{2}}} K\left(k_{1}\right)+2 \frac{\theta\left[\left(W_{1}-W_{2}\right)^{2}-\left(\varepsilon-\tilde{\varepsilon}_{1}\right)^{2}\right]}{\sqrt{\left(W_{1}+W_{2}\right)^{2}-\left(\varepsilon-\tilde{\varepsilon}_{1}\right)^{2}}} K\left(k_{2}\right)\right\},
$$

где

$$
k_{1}^{2}=\frac{\left(W_{1}+W_{2}\right)^{2}-\left(\varepsilon-\tilde{\varepsilon}_{1}\right)^{2}}{4 W_{1} W_{2}}, \quad k_{2}^{2}=\frac{4 W_{1} W_{2}}{\left(W_{1}+W_{2}\right)^{2}-\left(\varepsilon-\tilde{\varepsilon}_{1}\right)^{2}},
$$

$K(k)$ - эллиптический интеграл первого рода. При $W_{1}=W_{2}=W, a=b$ мы получаем выражение для двумерной изотропной системы

$$
N(\varepsilon)=\frac{1}{\pi^{2} a^{2} W} K\left(k_{1}\right) .
$$

В предельном случае $W_{2}=0$ получаем выражение для квазиодномерной системы с корневой особенностью

$$
N(\varepsilon)=\frac{1}{\pi a \sqrt{W_{1}^{2}-\left(\varepsilon-\varepsilon_{1}\right)^{2}}} .
$$


В двумерном случае формула (20) имеет логарифмическую особенность (пик вблизи точки $\varepsilon=0$ при $\left.W_{1}=W_{2}\right)$, поскольку

$$
K \rightarrow \ln \frac{4}{\sqrt{1-k^{2}}} \quad \text { при } \quad k \rightarrow 1 .
$$

В случае $W_{1} \neq W_{2}$ в зависимости $N(\varepsilon)$ имеются две особые точки, что приводит к возникновению двух пиков, разделенных расстоянием $2\left(W_{1} / W_{2}-1\right)$ [24]. Эти особенности плотности электронных состояний, на наш взгляд, должны проявляться в рассматриваемых нами двумерных системах при введении примеси или под давлением. Как следует из работы [23], такого рода особенности четко проявляются в кинетических коэффициентах, таких, например, как проводимость и термо-ЭДС в материалах высокотемпературной сверхпроводимости.

Отметим три особенности плотности электронных состояний (20):

1) $N(\varepsilon)$ ограничивает область изменения $\varepsilon$ конечными пределами $\left[\varepsilon_{a}, \varepsilon_{b}\right]$, где $\left\{\begin{array}{l}\varepsilon_{a} \\ \varepsilon_{b}\end{array}\right\}=\tilde{\varepsilon}_{1} \mp\left(W_{1}+W_{2}\right)$;

2) $N(\varepsilon)$ имеет логарифмические расходимости в точках $\varepsilon_{c}, \varepsilon_{d}$ где $\left\{\begin{array}{l}\varepsilon_{c} \\ \varepsilon_{d}\end{array}\right\}=\varepsilon_{1} \mp$ $\left(W_{1}-W_{2}\right)$

$3)$ первое слагаемое в (20) отлично от нуля на отрезках $\left[\varepsilon_{a}, \varepsilon_{c}\right]$ и $\left[\varepsilon_{d}, \varepsilon_{b}\right]$, а второе слагаемое - на отрезке $\left[\varepsilon_{c}, \varepsilon_{d}\right]$.

В соответствии с определениями (16) и (17) введем интегралы

$$
C_{i}\left(\mu_{\beta}^{\alpha}, \Delta, M\right)=\int_{\varepsilon_{a}}^{\varepsilon_{b}} d \varepsilon N(\varepsilon) \Phi_{i}\left(\varepsilon, \mu_{\beta}^{\alpha}, M, \Delta\right)=\mathrm{I}+\mathrm{II}, \quad i=1,2,
$$

где I - вклад от первого члена в плотность электронных состояний,

$$
\mathrm{I}=\int_{\varepsilon_{a}}^{\varepsilon_{c}} d \varepsilon N(\varepsilon) \Phi_{i}\left(\varepsilon, \mu_{\beta}^{\alpha}, \Delta, M\right)+\int_{\varepsilon_{d}}^{\varepsilon_{b}} d \varepsilon N(\varepsilon) \Phi_{i}\left(\varepsilon, \mu_{\beta}^{\alpha}, \Delta, M\right),
$$

а II - вклад от второго члена выражения (20),

$$
\mathrm{II}=\int_{\varepsilon_{c}}^{\varepsilon_{1}} d \varepsilon N(\varepsilon) \Phi_{i}\left(\varepsilon, \mu_{\beta}^{\alpha}, \Delta, M\right)+\int_{\varepsilon_{1}}^{\varepsilon_{d}} d \varepsilon N(\varepsilon) \Phi_{i}\left(\varepsilon, \mu_{\beta}^{\alpha}, \Delta, M\right) .
$$

Перейдем в выражении (26) к новой переменной $x^{2}=\left(W_{1}+W_{2}\right)^{2}-\left(\varepsilon-\varepsilon_{1}\right)^{2} / 4 W_{1} W_{2}$ и приведем его к виду

$$
\mathrm{I}=\int_{0}^{1} d x K(x) x \Psi_{i}\left(x, \mu_{\beta}^{\alpha}, \Delta, M\right) 4 \sqrt{W_{1} W_{2}},
$$

где $K(x)$ - эллиптический интеграл первого рода,

$$
\begin{aligned}
& \Psi_{i}\left(x, \mu_{\beta}^{\alpha}, \Delta, M\right)=\left[\Phi_{i}\left(\tilde{\varepsilon}_{1}-\sqrt{\left(W_{1}+W_{2}\right)^{2}-4 W_{1} W_{2} x^{2}}, \mu_{\beta}^{\alpha}, \Delta, M\right)+\right. \\
& \left.\quad+\Phi_{i}\left(\tilde{\varepsilon}_{1}+\sqrt{\left(W_{1}+W_{2}\right)^{2}-4 W_{1} W_{2} x^{2}}, \mu_{\beta}^{\alpha}, \Delta, M\right)\right]\left[\left(W_{1}+W_{2}\right)^{2}-4 W_{1} W_{2} x^{2}\right]^{-1 / 2} .
\end{aligned}
$$

В выражении для II перейдем к новой переменной $x^{2}=4 W_{1} W_{2} /\left[\left(W_{1}+W_{2}\right)^{2}-\right.$ $\left.\left(\varepsilon-\tilde{\varepsilon}_{1}\right)^{2}\right]$ и приведем $(27)$ к виду

$$
\mathrm{II}=\int_{2 \sqrt{\eta} /(1+\eta)}^{1} d x K(x) \frac{1}{x^{2}} \Psi_{i}\left(\frac{1}{x}, \mu_{\beta}^{\alpha}, \Delta, M\right) 4 \sqrt{W_{1} W_{2}} .
$$


В результате приведенного выше учета областей интегрирования по энергии (I и II), в которых плотность электронных состояний отлична от нуля, систему уравнений (16) и (17) можно привести к виду

$$
\begin{aligned}
\frac{1}{N_{0} V}= & \sqrt{W_{1} W_{2}} \sum_{\alpha, \beta} \int_{0}^{1} d x K(x) x \Psi_{1}\left(x, \mu_{\beta}^{\alpha}, \Delta, M\right)+ \\
& +\sqrt{W_{1} W_{2}} \sum_{\alpha, \beta} \int_{2 \sqrt{\eta} /(1+\eta)}^{1} \frac{K(x) d x}{x^{2}} \Psi_{1}\left(\frac{1}{x}, \mu_{\beta}^{\alpha}, \Delta, M\right), \\
\frac{1}{N_{0} I}= & \sqrt{W_{1} W_{2}} \sum_{\alpha, \beta} \int_{0}^{1} d x x K(x) \Psi_{2}\left(x, \mu_{\beta}^{\alpha}, \Delta, M\right)+ \\
& +\sqrt{W_{1} W_{2}} \sum_{\alpha, \beta} \int_{2 \sqrt{\eta} /(1+\eta)}^{1} \frac{d x}{x^{2}} K(x) \Psi_{2}\left(\frac{1}{x}, \mu_{\beta}^{\alpha}, \Delta, M\right),
\end{aligned}
$$

где $\eta=W_{2} / W_{1}, N_{0}=1 / \pi^{2} a b W_{1}$. Система уравнений (31) определяет значения параметров порядка $\Delta$ и $M$ в фазе сосуществования сверхпроводимости и состояния ВСП. В таком виде ее удобно использовать при выполнении численных расчетов.

\section{4. КРИТИЧЕСКАЯ ТЕМПЕРАТУРА МАГНИТНОГО И СВЕРХПРОВОДЯЩЕГО УПОРЯДОЧЕНИЯ}

Для понимания значения учета процессов переброса удобно рассмотреть сначала переход квазидвумерной системы в магнитную фазу $T_{\mathrm{m}}>T_{\mathrm{c}}\left(T_{\mathrm{m}}\right.$ и $T_{\mathrm{c}}$ - температура перехода в состояние ВСП и в сверхпроводящее состояние соответственно).

Полагая в формуле (17) $\Delta=0$ и $M=0$, получаем уравнение для определения величины $T_{\mathrm{m}}$ :

$$
\frac{1}{I}=\frac{1}{2} \sum_{\alpha, \beta} \int_{0}^{\widetilde{W}} \frac{d \varepsilon}{\varepsilon} N(\varepsilon)\left[\operatorname{th} \frac{\varepsilon+\mu_{\beta}^{\alpha}}{2 T_{\mathrm{m}}}+\operatorname{th} \frac{\varepsilon-\mu_{\beta}^{\alpha}}{2 T_{\mathrm{m}}}\right],
$$

где $N(\varepsilon)$ и $\mu_{\beta}^{\alpha}$ определяются формулами (20) и (15) соответственно, $\alpha, \beta= \pm 1$. Необходимо определить параметры $\eta_{a}$ и $\eta_{b}$. С этой целью мы налагаем условия $d T_{\mathrm{m}} / d \eta_{a}=0$ и $d T_{\mathrm{m}} / d \eta_{b}=0$, что соответствует максимальному значению температуры магнитного перехода (минимуму свободной энергии). Эти условия приводят к двум дополнительным уравнениям. Первое из них имеет вид

$$
\int_{0}^{\widetilde{W}} N(\varepsilon) \frac{d \varepsilon}{\varepsilon} \sum_{\alpha, \beta} \alpha\left\{\left[\operatorname{ch}^{2} \frac{\varepsilon+\mu_{\beta}^{\alpha}}{2 T_{\mathrm{m}}}\right]^{-1}-\left[\operatorname{ch}^{2} \frac{\varepsilon-\mu_{\beta}^{\alpha}}{2 T_{\mathrm{m}}}\right]^{-1}\right\}=0 .
$$

Второе уравнение получим из (33) заменой $\alpha \leftrightarrow \beta$. В дальнейшем мы несколько упрощаем эти уравнения, полагая, что $W_{1}$ и $W_{2}$ - величины одного порядка, и в нулевом приближении используем функцию $N(\varepsilon)$ изотропной системы $(22)$, которую можно представить в виде

$$
N(\varepsilon)=N_{0} \ln \frac{|\varepsilon|}{8 W_{1}} .
$$

Результаты численного решения системы уравнений (32), (33) определяются параметром $\mu$ - значением отклонения от серединного заполнения энергетической зоны: 


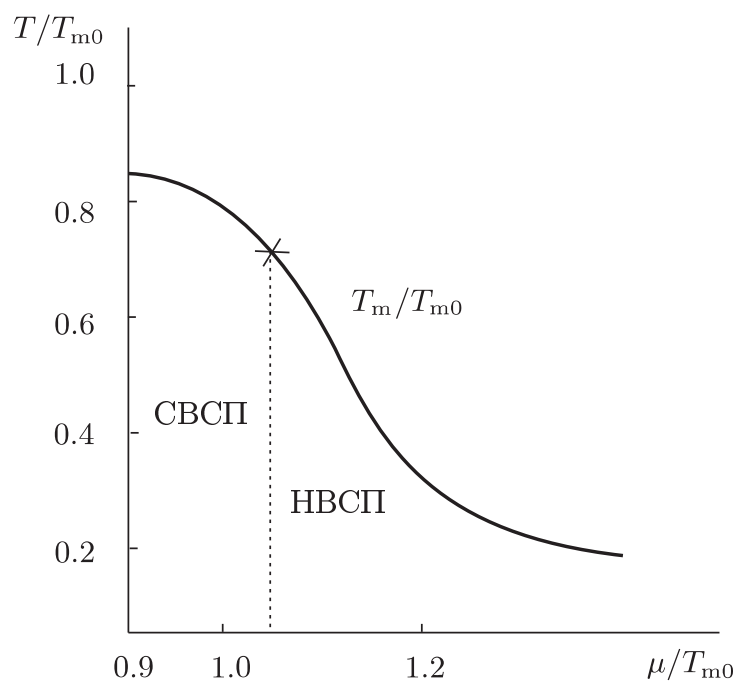

Рис. 1. Фазовая диаграмма $(T, \mu)$. СВСП и НВСП - соизмеримое и несоизмеримое состояния ВСП соответственно.

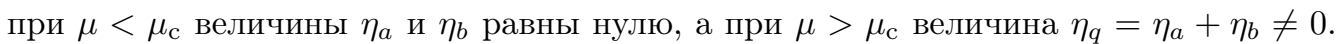
Величины $\eta_{a}$ и $\eta_{b}$ можно рассматривать как компоненты параметра порядка несоизмеримой фазы ВСП. Наличие величины $\eta_{q}$ говорит о переходе системы в полуметаллическое состояние из-за смещения диэлектрической щели относительно уровня Ферми $(q \neq 0)$ и возникновении свободных носителей заряда.

На рис. 1 представлена зависимость температуры магнитного перехода $T_{\mathrm{m}}$ как функция от $\mu$. Крестик на этой кривой определяет координаты $\left(T_{\mathrm{m}}, \mu_{\mathrm{c}}\right)$, отвеча-

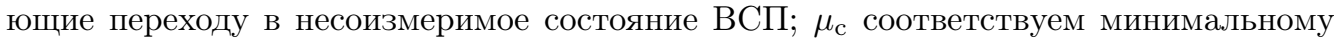
значению $\mu$, при котором возникает решение системы уравнений (32), (33) с $\eta_{q} \neq 0$.

В настоящей работе мы рассматриваем фиксированное значение $\mu$, что соответствует наличию в системе зоны, которая служит резервуаром. Существенное влияние на величину $\mu$ оказывает легирование. Детальное исследование влияния легирования на свойства двумерной магнитной системы с изотропным энергетическим спектром приведено в работе [22].

Наличие двух компонент параметра порядка $\eta_{a}$ и $\eta_{b}$, имеющее место в анизотропных системах, позволяет сделать вывод о принципиальной возможности структурного перехода.

Из формул (15) вытекает

$$
\frac{a-b}{a+b}=\frac{W_{2} q_{y} \eta_{a}-W_{1} q_{x} \eta_{b}}{W_{2} q_{y} \eta_{a}+W_{1} q_{x} \eta_{b}} .
$$

Под влиянием примеси или давления параметры, входящие в правую часть соотношения (35), меняются, что может привести к равенству $a=b$. Это значит, что система из орторомбической структуры $(a \neq b)$ переходит в тетрагональную фазу $(a=b)$. 
Рассмотрим зарождение сверхпроводимости на фоне магнитной фазы. Полагая в системе уравнений $(16),(17) M \neq 0, \Delta=0$, получаем

$$
\begin{gathered}
\frac{1}{V}=\frac{1}{2} \int_{0}^{\widetilde{W}} \frac{N(\varepsilon) d \varepsilon}{\sqrt{\varepsilon^{2}+M^{2}}} \sum_{\alpha, \beta, j} \frac{\left(M^{2}+j \mu_{\beta}^{\alpha} \sqrt{\varepsilon^{2}+M^{2}}\right) \operatorname{th} \frac{\sqrt{\varepsilon^{2}+M^{2}}+j \mu_{\beta}^{\alpha}}{2 T}}{j \mu_{\beta}^{\alpha}\left(\sqrt{\varepsilon^{2}+M^{2}}+j \mu_{\beta}^{\alpha}\right)}, \\
\frac{1}{I}=\frac{1}{2} \int_{0}^{\widetilde{W}} \sum_{\alpha, \beta, j} \frac{N(\varepsilon) d \varepsilon}{\sqrt{\varepsilon^{2}+M^{2}}} \operatorname{th} \frac{\sqrt{\varepsilon^{2}+M^{2}}+j \mu_{\beta}^{\alpha}}{2 T} .
\end{gathered}
$$

Эта система уравнений определяет критическую температуру перехода магнитной системы с параметром $\mu \neq 0$ в сверхпроводящее состояние. Исследуем ее в двух предельных случаях: $T_{\mathrm{c}} \approx T_{\mathrm{m}}$ и $T_{\mathrm{c}} \ll T_{\mathrm{m}}$. В случае $T_{\mathrm{c}} \approx T_{\mathrm{m}}(M \rightarrow 0)$ на основании (36) и (37) и полагая $T=T_{\mathrm{c}}$, получаем

$$
\frac{1}{V}-\frac{1}{I}=\frac{1}{2} \int_{0}^{\widetilde{W}} \sum_{\alpha, \beta, j} \frac{N(\varepsilon) d \varepsilon}{\varepsilon} \operatorname{th} \frac{\varepsilon+j \mu_{\beta}^{\alpha}}{2 T_{\mathrm{c}}}\left[\frac{\varepsilon}{\varepsilon+j \mu_{\beta}^{\alpha}}-1\right] .
$$

При $\mu=0\left(\mu_{\beta}^{\alpha}=0\right)$ это условие выполняется только при $V=I$, т. е. когда затравочные параметры $T_{\mathrm{c} 0}=T_{\mathrm{m} 0}$, а при $\mu_{\beta}^{\alpha} \neq 0$ должно выполняться соотношение $1 / V>1 / I$, т. е. $T_{\mathrm{c} 0}<T_{\mathrm{m} 0}$. Нетрудно видеть, что правая часть уравнения (32) меньше нуля при $\mu_{\beta}^{\alpha}>0$. Мы получаем, что в случае $T_{\mathrm{c}} \approx T_{\mathrm{m}}$ в магнитной фазе сверхпроводимость возможна только в случае $T_{\mathrm{c} 0}<T_{\mathrm{m} 0}$. Кроме того, при малых $M$ $\left(M \ll T_{\mathrm{c}}\right)$ выполним разложение в соотношении $(36)$ по величине $\left(M / T_{\mathrm{c}}\right)^{2}$. В peзультате получаем

$$
\frac{1}{V}=F_{0}\left(\mu T_{\mathrm{c}}\right)+\left.M^{2} \frac{\partial}{\partial M^{2}} F\left(\mu, T_{\mathrm{c}}, M\right)\right|_{M=0}+\cdots,
$$

где $F\left(\mu, T_{\mathrm{c}}, M\right)$ - правая часть $(36)$, а $F_{0}\left(\mu, T_{\mathrm{c}}\right)=\left.F\left(\mu, T_{\mathrm{c}}, M\right)\right|_{M=0}$. Величина $T_{\mathrm{c}}$ убывает с ростом $M$. Коэффициент разложения при $M^{2}$ в выражении (39) зависит

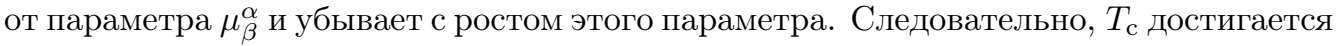
при $\mu>\mu_{\mathrm{c}}$ и растет с ростом параметра $\mu$.

При возникновении слабой сверхпроводимости на фоне магнитной фазы $T_{\mathrm{c}} \ll M$ следует рассматривать формулы (36) и (37). По-видимому, из-за сложного вида плотности электронных состояний (11) вычисления в этих формулах возможны численными методами. Аналитически можно выполнить оценки величины $T_{c}$, если полагать $N(\varepsilon)=N_{0}=$ const. Эти оценки будут качественно соответствовать рассмотренному квазиодномерному случаю [6], [7], [21] и в целом описывать ситуацию для изотропной системы. В этом случае $\left(T_{\mathrm{c}} \ll T_{\mathrm{m}}\right)$ поведение правых частей уравнений $(36),(37)$ зависит от соотношения между параметром порядка $M$ и величинами $\mu_{\beta}^{\alpha}$. Можно сделать следующие выводы.

1. При $\mu_{-}^{-}<\mu_{-}^{+}, \mu_{+}^{+}<M$ сверхпроводимость не возникает на фоне ВСП. Этот результат противоположен случаю пайерлсовского перехода [19], [20], когда при аналогичном соотношении между приведенными выше параметрами возможно куперовское упорядочение.

2. При $\mu_{-}^{-}<M<\mu_{+}^{+}$, а также при $M<\mu_{-}^{-}<\mu_{+}^{+}$переход рассматриваемой системы в сверхпроводящее состояние оказывается возможным. В этом случае выраже-

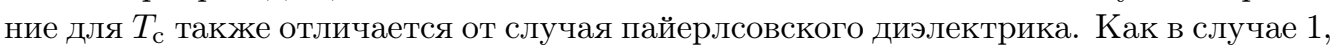


так и в случае 2 условия возникновения сверхпроводимости в магнитных системах являются более жесткими, чем в пайерлсовских диэлектриках.

3. Рассмотрим обратный случай, а именно $T_{\mathrm{c}}>T_{\mathrm{m}}$. Анализ системы уравнений (16) и (17) при $T=T_{\mathrm{m}}(M=0, \Delta \neq 0)$ показывает, что эти уравнения не дают решений, характерных для перехода в магнитное состояние. Таким образом, если рассматриваемая система находится в сверхпроводящем состоянии, то переход в магнитное состояние при понижении температуры невозможен. В дальнейшем мы будем рассматривать случай $T_{\mathrm{m}}>T_{\mathrm{c}}$.

\section{5. СВОБОДНАЯ ЭНЕРГИЯ СИСТЕМЫ В ФАЗЕ СОСУЩЕСТВОВАНИЯ МАГНЕТИЗМА И СВЕРХПРОВОДИМОСТИ. СКАЧОК ТЕПЛОЕМКОСТИ ПРИ $T=T_{\mathrm{c}}$}

Для определения свободной энергии воспользуемся тождеством

$$
F(\Delta, M)-F(0,0)=\int_{0}^{\Delta} \Delta^{\prime 2} d \Delta^{\prime} \frac{d}{d \Delta^{\prime}}\left(\frac{1}{V}\right)+\left.\int_{0}^{M} M^{\prime 2} d M^{\prime} \frac{d}{d M^{\prime}}\left(\frac{1}{I}\right)\right|_{V=0} .
$$

Выполнив в этой формуле интегрирование по частям, представим ее в виде

$$
F(\Delta, M)-F(0,0)=\frac{\Delta^{2}}{V}+\frac{M^{2}}{I}-2 \int_{0}^{\Delta} \Delta^{\prime} d \Delta^{\prime} \frac{1}{V}-\left.2 \int_{0}^{M} M^{\prime} d M^{\prime}\left(\frac{1}{I}\right)\right|_{V=0} .
$$

Подставляя в (40) вместо $1 / V$ и вместо $\left.(1 / I)\right|_{M=0}$ выражения (16) и (17) соответственно и выполнив интегрирования по параметрам порядка $\Delta^{\prime}$ и $M^{\prime}$, приведем разность свободных энергий к виду

$$
\begin{aligned}
F(\Delta, M)-F(0,0)= & \frac{\Delta^{2}}{V}+\frac{M^{2}}{I}-T \int_{-\widetilde{W}}^{\widetilde{W}} N(\varepsilon) d \varepsilon \times \\
& \times \sum_{\alpha, \beta}\left[\ln \operatorname{ch} \frac{\sqrt{X_{1}}}{2 T}+\ln \operatorname{ch} \frac{\sqrt{X_{2}}}{2 T}-\ln \operatorname{ch} \frac{\sqrt{X_{1}^{00}}}{2 T}-\ln \operatorname{ch} \frac{\sqrt{X_{2}^{00}}}{2 T}\right],
\end{aligned}
$$

где $X_{1}$ и $X_{2}$ определяются формулой (18), а $X_{1,2}^{00}=\left.X_{1,2}\right|_{M=\Delta=0}$. Из выражения для свободной энергии (42) нетрудно получить систему уравнений $(16),(17)$ для параметров порядка $\Delta$ и $M$. Для этого следует рассмотреть производные

$$
\frac{\partial F(\Delta, M)}{\partial \Delta^{2}}=0, \quad \frac{\partial F(\Delta, M)}{\partial M^{2}}=0 .
$$

Согласно результатам раздела 4 в рассматриваемой системе сверхпроводимость зарождается в фазе магнитного упорядочения. Следовательно, имеет смысл иследовать разность свободных энергий вида

$$
\begin{gathered}
\delta F=F(\Delta, M)-F(0, M)=\frac{\Delta^{2}}{V}-T \sum_{\alpha, \beta} \int_{-\widetilde{W}}^{\widetilde{W}} N(\varepsilon) d \varepsilon \times \\
\times \sum_{\alpha, \beta}\left[\frac{\ln \operatorname{ch} \sqrt{X_{1}}}{2 T}+\ln \operatorname{ch} \frac{\sqrt{X_{2}}}{2 T}-\ln \operatorname{ch} \frac{\sqrt{X_{1}^{0}}}{2 T}-\ln \operatorname{ch} \frac{\sqrt{X_{2}^{0}}}{2 T}\right], \\
X_{1}^{0}=\left.X_{1}\right|_{\Delta=0}, \quad X_{2}^{0}=\left.X_{2}\right|_{\Delta=0 .}
\end{gathered}
$$


В области температур, близких к температуре сверхпроводящего перехода $\left(T \sim T_{\mathrm{c}}\right)$, разложим разность свободных энергий (43) по малому параметру $\Delta^{2}$ :

$$
\delta F=F(\Delta, M)-F(0, M)=\frac{N_{0} \Delta^{4}}{4}\left(-f_{1}+\Delta^{2} f_{2}+\cdots\right),
$$

а также получим уравнение для температуры сверхпроводящего перехода (16)

$$
\frac{1}{V}=f_{0}-\frac{1}{2} f_{1} \Delta^{2}+\frac{3}{8} \Delta^{4} f_{2}+\cdots,
$$

где

$$
\begin{aligned}
& f_{0}=\left.\frac{1}{2} \int_{0}^{\widetilde{W}} d \varepsilon N(\varepsilon) \sum_{\alpha, \beta} \Phi_{1}\left(\varepsilon, \mu_{\beta}^{\alpha}, M, \Delta\right)\right|_{\Delta=0}, \\
& f_{1}=-\left.\int_{0}^{\widetilde{W}} d \varepsilon N(\varepsilon) \sum_{\alpha, \beta} \frac{\partial}{\partial \Delta^{2}} \Phi_{1}\left(\varepsilon, \mu_{\beta}^{\alpha}, M, \Delta\right)\right|_{\Delta=0}, \\
& f_{2}=\left.\frac{2}{3} \int_{0}^{\widetilde{W}} d \varepsilon N(\varepsilon) \sum_{\alpha, \beta} \frac{\partial^{2}}{\partial \Delta^{4}} \Phi_{1}\left(\varepsilon, \mu_{\beta}^{\alpha}, M, \Delta\right)\right|_{\Delta=0} .
\end{aligned}
$$

Как видно из выражения (44), имеются две возможности перехода магнитной системы в сверхпроводящее состояние: 1) $\Delta=0,2) \Delta^{2}=f_{1} / f_{2}$. Первый случай соответствует фазовому переходу второго рода (при $f_{1}<0$ ), а второй - фазовому переходу первого рода (при $\left.f_{1}>0\right)$. Решающее значение здесь имеет знак коэффициента $f_{1}$, зависящего от $\mu$ и $M$. Точка $f_{1}=0$ соответствует значению $\mu=\mu^{\prime}$, при котором меняется тип фазового перехода. Критическая температура не является монотонно убывающей однозначной функцией от $\mu$. Имеется область неоднозначного соответствия между $\mu$ и $T_{\mathrm{c}}$. Это область нестабильности нормального состояния по отношению к образованию куперовских пар. Очевидно, при $\mu>\mu^{\prime}$ следует рассматривать уравнение (45) при $\Delta \neq 0$, что соответствует фазовому переходу первого

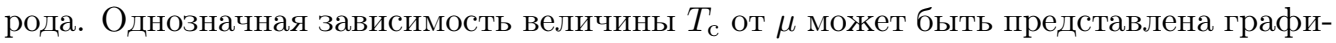
чески после вычисления разности свободных энергий в области низких температур по формуле (43) и определения величины $\mu$ из условия $\delta F=0$ при $T=T_{\mathrm{c}}=0$.

\section{6. ОБСУЖДЕНИЕ РЕЗУЛЬТАТОВ И ВЫВОДЫ}

В настоящей работе изучены термодинамические свойства слоистой системы с анизотропным энергетическим спектром и отклонением от серединного заполнения энергетической зоны $(\mu \neq 0)$. В такой системе происходит нарушение условия нестинга, т. е. $\varepsilon(\vec{k}) \neq-\varepsilon(\vec{k}+\vec{Q})$. Диэлектрическая щель смещается по отношению к уровню Ферми из-за процессов переброса, и возникают свободные носители заряда. В результате диэлектрик становится полуметаллом и на фоне магнетизма возможно возникновение сверхпроводимости. На наш взгляд, этот механизм может работать в диэлектриках и полупроводниках, полученных в последние годы и, в частности, связанных со слоистой структурой, в основе которой лежат атомы Fе.

Мы рассмотрели переход в несоизмеримое состояние ВСП и возможность возникновения сверхпроводимости в такой системе. 
На основании гамильтониана (1) получены система уравнений для функций Грина (5) и, следовательно, система уравнений для параметров сверхпроводящего и магнитного порядков. После выполнения в этих уравнениях суммирования по мацубаровской частоте выполнен переход к интегрированию по энергии в соответствии с методикой, позволяющей ввести параметры $\mu_{\beta}^{\alpha}=\mu+\alpha \eta_{a}+\beta \eta_{b}(\alpha, \beta= \pm 1)$, ответственные за возникновение несоизмеримого состояния ВСП. Наряду с этим вводится плотность электронных состояний (20), отвечающая закону дисперсии энергии электронов (11).

На наш взгляд, представляет интерес разработка методики, позволяющей учитывать при выполнении интегрирования по энергии только те области, где плотность электронных состояний $N(\varepsilon)(20)$ отлична от нуля. Эта методика позволяет выбрать конечные пределы интегрирования по энергии (см. (20)-(25)).

Сосуществование сверхпроводимости и состояния ВСП определяется условиями $\Delta \neq 0$ и $M \neq 0$, что отвечает нетривиальному решению системы уравнений $(16),(17)$. Наряду с этим необходимо, чтобы разность свободных энергий $\delta F=F(\Delta, M)$ $F(0, M)$ была отрицательной величиной, что соответствует выгодности зарождения сверхпроводимости на фоне магнитного состояния. Указанная выше система уравнений в представленном виде может быть решена только численными методами. Однако мы изучили некоторые предельные случаи, позволяющие выполнить аналитические исследования.

Важную роль в формировании поведения рассматриваемой системы имеет соотношение между константами электронно-фононного $V$ и магнитного $I$ взаимодействий (между затравочными значениями $T_{\mathrm{c} 0}$ и $T_{\mathrm{m} 0}$ ). В первую очередь был рассмотрен фазовый переход системы в состояние ВСП. В этом случае необходимо исследовать систему уравнений $(29)$, (30), численное решение которой позволяет получить значения температуры магнитного перехода $T_{\mathrm{m}}$, а также параметров $\eta_{a}$ и $\eta_{b}$, которые оказываются отличными от нуля при некотором $\mu>\mu_{\mathrm{c}}$. Условия $\eta_{a} \neq 0$, $\eta_{b} \neq 0$ соответствуют переходу системы в несоизмеримое состояние ВСП, эти величины играют роль параметров порядка этой фазы. Зависимости $T_{\mathrm{m}}$ и $\eta_{q}=\eta_{a}+\eta_{b}$ от $\mu$ приведены на рис. 1 и рис. 2 соответственно. Отметим, что условия $\eta_{a} \neq 0$ и $\eta_{b} \neq 0$ понижают энергию системы и стабилизируют состояние ВСП, которое перестает быть диэлектрическим состоянием. На фоне этого состояния возможно возникновение сверхпроводимости. Рассмотрены два предельных случая: $T_{\mathrm{c}} \approx T_{\mathrm{m}}$ и $T_{\mathrm{c}} \ll T_{\mathrm{m}}$.

В первом случае критическая температура $T_{\text {c }}$ существенно зависит от парамет-

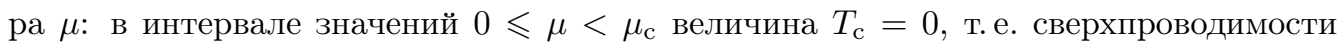
не возникает, а при $\mu>\mu_{\mathrm{c}}$ сверхпроводимость имеет место. Величина $T_{\mathrm{c}}$ растет с уменьшением параметра порядка $M$. Во втором случае сверхпроводимость возникает при условии, что величина $M$ меньше хотя бы одного из значений параметров $\mu_{\beta}^{\alpha}$ $(\alpha, \beta= \pm 1)$. В случае же $\mu_{\beta}^{\alpha}<M$ зарождение сверхпроводимости на фоне состояния ВСП невозможно. Если предположить, что система находится в сверхпроводящем состоянии $T_{\mathrm{c}}>T_{\mathrm{m}}$, то положив в уравнении $T=T_{\mathrm{m}}, M=0$ и $\Delta \neq 0$, нетрудно видеть из (17), что в такой сверхпроводящей системе магнетизм не возникает.

Отметим, что полученное выражение для скачка теплоемкости $\left(C_{\mathrm{S}}-C_{\mathrm{N}}\right) / N_{0}$ при

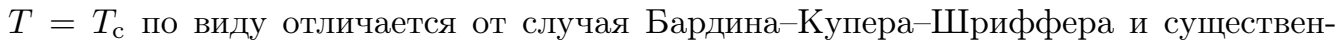
но зависит от параметров $\mu$ и $M$. В развитой выше теории сверхпроводимости 


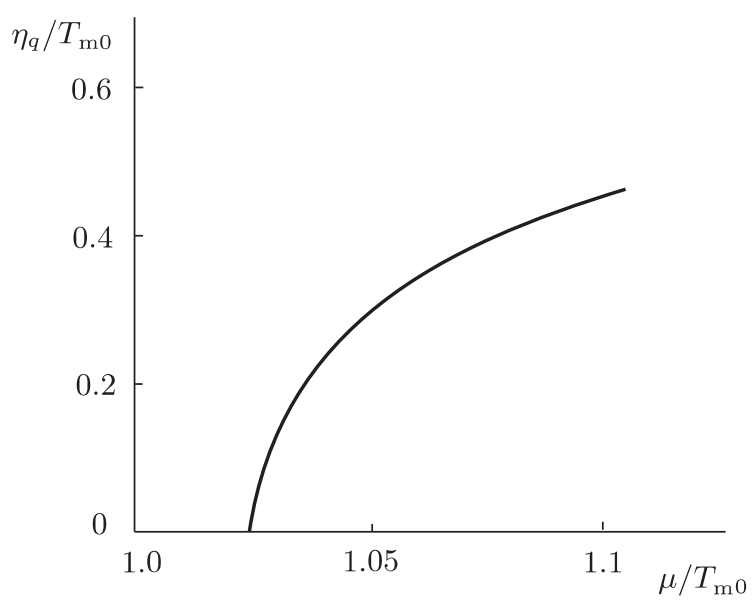

Рис. 2. Зависимость параметра $\eta_{q}$ от $\mu$ в точке перехода металл-диэлектрик $\left(T=T_{\mathrm{m}}\right)$.

и магнетизма фигурирует плотность электронных состояний, содержащая параметры $W_{1}, W_{2}$, а также постоянные решетки $a, b$. Это обстоятельство позволяет в полной мере учитывать влияние примеси и давления (через изменения этих параметров) на величины $T_{\mathrm{m}}, T_{\mathrm{c}},\left.\left(\left(C_{\mathrm{S}}-C_{\mathrm{N}}\right) / N_{0}\right)\right|_{T=T_{\mathrm{c}}}$, а также на возможность структурного порядка.

\section{Список литературы}

[1] М. В. Садовский, УФН, 178:12 (2008), 1243-1271; arXiv: 0812.0302.

[2] Y. Kamihara, T. Watanabe, M. Hirano, H. Hosono, J. Am. Chem. Soc., 130:11 (2008), 3296-3297.

[3] K. Ishida, Y. Nakai, H. Hosono, J. Phys. Jpn., 78 (2009), 062001, 20 pp., arXiv: 0906.2045.

[4] X. Zhu, H. Yang, L. Fang, G. Mu, H.-H. Wen, Supercond. Sci. Technol., 21:10 (2008), 105001, 5 pp.

[5] В. А. Москаленко, М. Е. Палистрант, В. М. Вакалюк, УФН, 161:8 (1991), 155-178.

[6] В. А. Москаленко, Л. З. Кон, М.Е. Палистрант, Низкотемпературные свойства металлов с особенностями зонного спектра, Штиинца, Кишинев, 1989.

[7] V. A. Moscalenko, L.Z. Kon, M. E. Palistrant, Theory of the Multiband Superconductivity, Tehnica, Bucharest, 2008 (in Romanian).

[8] M. E. Palistrant, Condens. Matter Phys., 12:1 (2009), 677-688.

[9] M. E. Palistrant, L. Z. Kon, Ukr. J. Phys., 55:1 (2010), 44-54.

[10] V. Barzykin, L. P. Gor'kov, Писъма в ЖКЭТФ, 88:2 (2008), 142-146.

[11] Ė.Z. Kuchinskii, M. V. Sadovskii, Писъма в ЖЭЭТФ, 89:3 (2009), 176-180.

[12] L. Benfatto, M. Capone, S. Caprara, C. Castellani, C. Di Castro, Phys. Rev. B, 78:14 (2008), 140502(R), 4 pp., arXiv: 0807.4408.

[13] M. G. Vavilov, A. V. Chubukov, A. B. Vorontsov, Supercond. Sci. Technol., 23:5 (2010), 054011, arXiv: 0912.3556.

[14] E. E. Rodriguez, C. Stock, K. Krycka, C.F. Majkrzak, K. Kirshenbaum, N.P. Butch, S. R. Shanta, J. Paglione, M. A. Green, Phys. Rev. B, 83:13 (2011), 134438, 6 pp., arXiv: 1012.5311 . 
[15] С. В. Тябликов, Методы квантовой теории магнетизма, Наука, М., 1965.

[16] В. Л. Бонч-Бруевич, С. В. Тябликов, Метод функций Грина в статистической механике, Физматлит, М., 1961.

[17] A. B. Vorontsov, M. G. Vavilov, A. V. Chubukov, Phys. Rev. B, 81 (2010), 174538, 17 pp., arXiv: 1003.2389 .

[18] M. K. Forthaus, K. Sengupta, O. Heyer, N.E. Christensen, A. Svane, K. Syassen, D. I. Khomskii, T. Lorenz, M. M. Abd-Elmeguid, Phys. Rev. Lett., 105:15 (2010), 157001, 5 pp., arXiv: 1009.3787.

[19] M. C. Leung, Phys. Rev. B, 11:11 (1975), 4272-4277.

[20] Ф.Х. Тимеров, ЖЭЭТФ, 72:6 (1977), 2309-2323.

[21] М. Е. Палистрант, И. В. Пэдуре, ТМФ, 62:1 (1985), 117-126; 70:3 (1987), 443-453;

M. E. Palistrant, I. V. Pédure, Phys. Lett. A, 111:8-9 (1983), 445-447.

[22] М.Е. Палистрант, В. М. Вакалюк, ФНТ, 18 (1992), 847.

[23] М.Е. Палистрант, Ф.Г. Кочорбэ, Изв. АН ССР Молдовы. Физика и техника, 2:5 (1991), 7.

[24] D. F. Digor, M. E. Palistrant, Mold. J. Phys. Sci., 9:3-4 (2010), 311.

Поступила в редакцию 1.03.2011 